\title{
From Hype to Reality: A Taxonomy of Blockchain Applications
}

\author{
Olga Labazova \\ University of Cologne \\ labazova@wiso.uni-koeln.de
}

\author{
Tobias Dehling \\ Karlsruhe Institute of Technology \\ dehling@kit.edu
}

\author{
Ali Sunyaev \\ Karlsruhe Institute of Technology \\ sunyaev@kit.edu
}

\begin{abstract}
Blockchain is a decentralized digital ledger that challenges existing business models and theories by shifting the trust from institutions towards algorithms. However, the number of successfully developed blockchain-based systems remains low. This points towards a research gap between blockchain applications and technical blockchain characteristics. We answer the research question: What application areas fit blockchains with what technical characteristics? We develop a taxonomy, which comprises six blockchain application areas that are classified across eight technical dimensions. We demonstrate the utility of the taxonomy on ninetynine blockchain-based systems. We contribute to the scientific literature by delimiting blockchain application areas, identifying new technical dimensions, and linking application and technical knowledge on blockchain to guide development of blockchain-based systems. For practitioners, we present an overview of current blockchain-based systems.
\end{abstract}

\section{Introduction}

A blockchain is a decentralized digital ledger [12] with the unique value proposition to shift the trust from institutions towards algorithms [25]. The future impact of blockchains on existing business models and theories might be comparable to the invention of smart phones or the internet $[3,9,27,41]$. Therefore, researchers and practitioners jump on the blockchain bandwagon $[1,3]$ in attempts to replace established trust-based business models with blockchains [12, 37]. The hype emerging around blockchains suggests that blockchains can replace banks in the financial sector [25, 37], support agreements among individuals or internet-of-things devices using smart contracts $[18,28]$, and manage essential records (e.g., health records, education records) that are currently maintained by centralized organizations $[2,32]$.
Yet, challenges of developing blockchain-based systems outweigh envisioned benefits [8]. Most of the current blockchain projects could not move from ideas to production use [13]. For example, projects aimed at employing blockchains to support tokenization of space missions (e.g., SpaceBIT) or artificial intelligence [35] did not reveal proofs of concept. Narrow-scoped blockchain prototypes experience issues with scalability of blockchain protocols, waste of computational resources required for consensus mechanisms, traceability of users, and a lack of network protection against fraud $[11,36,44$, 45]. Currently, practitioners continue experimenting with proofs of concept and system designs based on trial-and-error approaches [13].

Extant research in the blockchain domain is focused on the development of blockchain-based systems and the diversity of technical components (e.g., consensus mechanisms, permissioning) and applications (e.g., financial transactions, the internet of things). A closer examination of extant research reveals the diversity of blockchain application areas with no-size-fits-all technical blockchain characteristics [20, 39, 44]. For example, the Bitcoin network is untrusted and requires a secure proof-ofwork consensus mechanism [25] while a Hyperledger business network ensures trust and can employ lighter consensus mechanisms, such as practical Byzantine fault tolerance [19]. The relevant technical blockchain characteristics, however, remain abstract, fragmented, and scattered across applications.

More knowledge connecting technical blockchain characteristics and blockchain applications is crucial to provide the guidelines on development of successful blockchain-based systems. Trial-and-error development leads to unfulfilled expectations in blockchain-based systems and loss of investments. Therefore, we answer the research question: What application areas fit blockchains with what technical characteristics?

Taxonomies are used to organize knowledge in many fields (e.g., Darwin's classification of species in biology) $[4,10,24,31]$. We choose a taxonomy as the fundamental tool to organize knowledge on blockchains [26]. We develop a taxonomy of 
blockchain applications, which captures six blockchain application areas that are classified across eight technical dimensions [26]. The taxonomy is based on extant scientific literature, business reports, and previous blockchain classifications. We demonstrate the utility of the taxonomy by classifying ninety-nine blockchain-based systems [12, 43]. Extant blockchain taxonomies and other classifications describe blockchains from either technical or application perspectives [5, 17, 39, 44]. Our taxonomy is different because it integrates technical and application knowledge that allows to guide the development of blockchain-based systems.

This research contributes to the scientific knowledge base in three ways. First, we establish an overview of extant research on blockchain application areas. Second, we identify new technical dimensions of importance to blockchain applications, which complement extant work in the technical literature. Third, we link blockchain application areas and technical blockchain characteristics, which can guide development of blockchain-based systems. For practitioners, the taxonomy gives an overview of successful blockchain applications that can reduce development challenges for future blockchain-based systems.

This manuscript proceeds as follows. We start with related research on blockchain. Next, we outline the approach employed for taxonomy development. Then, we present the taxonomy of blockchain applications and demonstrate its utility on ninety-nine blockchain applications. Finally, we discuss principal findings, future research, limitations of our study, and implications for theory and practice.

\section{Related research}

The scientific literature on blockchain is at an early development stage. An absence of guidelines on development of blockchain-based systems hinders successful blockchain projects. Extant blockchain taxonomies and other classifications consider technical blockchain characteristics and blockchain application areas separately. Technical blockchain classifications are focused on the diversity of technical components (e.g., permissions to read transactions, consensus mechanisms) and cover predominantly the financial sector $[6,21,23,38,43$, 45]. For instance, a study comparing digital payment providers identifies permissions to read and write financial transactions as important technical characteristics to consider when choosing between centralized and decentralized payment platforms [21]. Centralized payment platforms give permissions on reading and writing financial transactions to authorized users; decentralized payment platforms do not require user authorization to read and write financial transactions. A review of cryptocurrencies investigates different consensus mechanisms, levels of anonymity, and data integrity among cryptocurrencies [23]. Different consensus mechanisms (e.g., proof-of-stake, practical Byzantine fault tolerance) are determined to be suitable to improve the efficiency of second-generation cryptocurrencies $[6,38,45]$. Compared to Bitcoin, Zerocoin guaranties a stronger anonymity of users that prevents user traceability $[11,14,29]$ and Litecoin has lower data integrity that allows for support of devices with low storage capacity (e.g., mobile phones) [15]. Further overviews of key technical characteristics of blockchains gather previous findings in the financial sector including reading and writing permissions of transactions, consensus mechanisms, anonymity levels, and other technical characteristics that are not focused on blockchain design but rather on interoperability (e.g., chain modularity) [16, 17, 39, 44].

Investigations of blockchain application areas start with the idea that blockchains can be useful beyond the financial sector. Extant research focuses predominantly on applying blockchains for digital payments, certification, cloud storage, identity management, energy distribution, and advanced tracking [30]. Business reviews of blockchain startups reveal new application areas including customer loyalty, cybersecurity, digital rights management, digital voting and government, gaming, content distribution, platform development, prediction markets, and smart contracts [12, 30].

Isolated knowledge of technical and application research causes hypes of blockchain application areas and technical blockchain characteristics. Further consideration and consolidation of application and technical knowledge on blockchains will result in a foundational classification of blockchain application areas in alignment with technical blockchain characteristics and provide the first steps to guide the development of successful blockchain-based systems.

\section{Research approach}

To organize knowledge on blockchains, we use the method for taxonomy development proposed by Nickerson et al., who define a taxonomy as a set of dimensions [26]. Each dimension consists of "mutually exclusive and collectively exhaustive characteristics in a way that each object under consideration has one and only one" [26:5] 
characteristic in every dimension. The taxonomy development method proceeds in three stages (Figure 1). In the initial stage, a metacharacteristic and ending conditions are defined according to the purposes of the taxonomy to be developed. In the main stage, the taxonomy is developed. Taxonomy objects (here application cases), dimensions, and characteristics are identified during inductive or deductive iterations. In inductive iterations, empirical cases are analyzed to determine dimensions and characteristics in the taxonomy. In deductive iterations, dimensions and characteristics are derived from the scientific knowledge base. In the final stage, the taxonomy is evaluated against ending conditions.

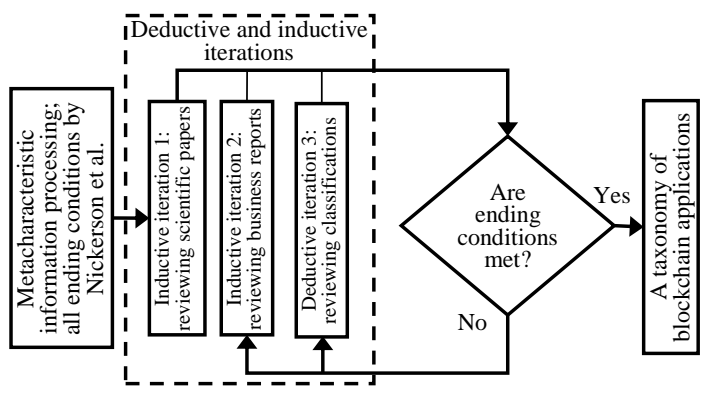

Figure 1. Research approach for development of the taxonomy of blockchain applications.

\subsection{Development of the taxonomy of blockchain applications}

The objective of the taxonomy is to classify blockchain application areas based on technical blockchain characteristics. Therefore, we selected technical blockchain characteristics (e.g., consensus mechanism, anonymity level) as the metacharacteristic. The choice and combination of technical blockchain characteristics are central to the success or failure of blockchain-based systems. The metacharacteristic serves as basis for identification of further dimensions and characteristics.

We developed the taxonomy in three iterations. The first two iterations were inductive iterations, where we identified application cases to derive dimensions and characteristics. For each inductive iteration, we used different types of sources: scientific literature and business reviews, respectively. The third iteration was a deductive iteration where we revised the taxonomy based on previous classifications. In the first iteration, we searched articles in the web of science core collection" with the search string "blockchain OR

\footnotetext{
${ }^{1}$ Used indices: "Science Citation Index Expanded (1900-present), Social Sciences Citation Index (1900-present), Arts \& Humanities
}

distributed ledger" on October 17, 2016, in title, abstract, and keywords, covering the whole period of publications [7, 40]. The search returned fifty-one papers. After screening of titles and abstracts, we coded the forty-one remaining relevant articles. In the first iteration, we identified six dimensions with fourteen characteristics and six application areas with sixteen application cases. The analysis of the scientific literature revealed detailed information on separate blockchain characteristics (e.g., consensus mechanisms) or specific blockchain application examples (e.g., energy markets, prediction platforms) but lacked comprehensiveness. In the second iteration, we analyzed business reviews, which provide less profound but more comprehensive information. We investigated twenty business reports by national agencies, consulting companies, and international institutions. We revised the taxonomy and added two dimensions, seven characteristics, and nine application cases. The third iteration was deductive, where we derived characteristics, dimensions, and application cases from fifteen previous classifications. We used all previous classifications that we could identify in extant literature until May 2018. Our taxonomy covers all characteristics in classifications related to technical blockchain characteristics.

All ending conditions proposed by Nickerson at al. [26] were fulfilled after the third iteration as follows. First, all found blockchain application cases described in the scientific literature or business reports can be classified into an application case in the taxonomy. Second, each dimension is unique and mutually exclusive, and each characteristic is unique within its dimension. Third, all application cases were classified with a single characteristic for each dimension. Fourth, the taxonomy is conciseconsists only of meaningful dimensions that classify application cases. Fifth, the taxonomy is robustdifferentiates each application case from all others. Sixth, the taxonomy is explanatory, comprehensive, and extensible - highlights the main features of each application case and can be extended when new application cases arise.

\subsection{Data analysis}

To analyze the sources, we used three types of coding: open coding, axial coding, and selective

Citation Index (1975-present), Conference Proceedings Citation Index- Science (1990-present), Conference Proceedings Citation Index- Social Science \& Humanities (1990-present), Book Citation Index-Science (2005-present), Book Citation Index-Social Sciences \& Humanities (2005-present), and Emerging Sources Citation Index (2015-present)" 
coding [33, 42]. Open coding is a process for grouping categories and subcategories [33:12]. Axial coding is a process for testing "that categories are related to their subcategories, and the relationships against data" [33:13]. Selective coding is a process "by which all categories are unified around a 'core' category, and categories that need further explication are filled-in with descriptive details" [33:14]. We applied open coding for initial categorization of dimensions, characteristics, application areas, and application cases; axial coding for removal of overlapping dimensions, characteristics, application areas, and application cases while iteratively testing the taxonomy against data; and selective coding to classify each application case with a characteristic for each dimension. One researcher coded the sources three times, in November 2016, April 2017, and November 2017, and other researchers validated the results after each iteration [34]. Disputes were resolved in group discussions.

\section{Taxonomy of blockchain applications}

The developed taxonomy consists of eight dimensions with twenty-one technical characteristics and six application areas with twenty-five application cases (Table 1).

\subsection{Technical blockchain characteristics}

The first dimension is reading access and represents different modes for reading information on blockchains. Private reading allows only authorized members to access a blockchain. Public reading access allows everyone to read data from a blockchain. The second dimension is writing access and represents different modes of writing information on a blockchain. Permissioned writing access requires users to be authorized to add transactions. If writing access is unpermissioned, a user does not have to be authorized to add transactions. The third dimension is main consensus mechanism and is concerned with employed means for updating blockchains; we focus on four predominant consensus mechanisms. Proof-of-work requires some resources (or work) from a requester, usually processing time of a computer to solve a computationally difficult puzzle. Proof-of-stake asks users to proof the ownership of a certain amount of digital data to establish their stake in this data. Practical Byzantine fault tolerance gathers individual decisions made by trusted nodes in a network that together determine system-level agreements. Selfdeveloped consensus mechanisms are used in some application cases and usually include several highly trusted nodes for arriving at system-level agreements. The fourth dimension is anonymity level and assesses whether users can be matched to identities. If blockchains have the characteristic anonymous, users do not have to provide any data to work with blockchains. If blockchains are pseudonymous, users have to work under a pseudonym. Blockchains with the characteristic identifiable ask for or automatically collect personally identifiable information, such as email addresses. The fifth dimension is event handling and discerns whether blockchains can handle application logic or events. No event handling shows an inability to handle application logic. Fixed event handling supports built-in events. Custom event handling means that a blockchain supports processing of any application logic provided by users. The sixth dimension is data exchange type that focuses on the type of information sharing between users on blockchains and includes the characteristics transaction and content. Transaction implies an exchange of logs of executed actions. Content means that digital assets, such as documents, messages, and video or music files, are exchanged. The seventh dimension is encryption and specifies whether data on blockchains is encrypted. Unencrypted means that no data on the blockchain is encrypted. Partiallyencrypted represents blockchain, where some data is encrypted. Totally-encrypted means that all data on blockchains is encrypted and has to be decrypted for all operations. The eighth dimension is history retention and ascertains whether the whole blockchain or only its recent updates are kept and distributed between hosts. Whole retention means that the whole history starting with a genesis block is kept in a blockchain and distributed between nodes. Recent updates retention specifies that only the latest updates are kept and distributed.

\subsection{Blockchain application cases}

We identified six blockchain application areas comprising a total of twenty-five application cases. Application areas capture the basic functionalities that can be performed by blockchains and group application cases with similar semantic features and similar combinations of technical blockchain characteristics. The first application area is financial transactions and captures seven application cases concerned with money transfer and exchange. Conventional cryptocurrencies use public unpermissioned blockchains, where consensus is achieved through proof-of-work, and users act under pseudonyms. Blockchains with the same characteristics except for anonymous user access 
support anonymous cryptocurrencies. To confirm interest of users in blockchain and to reduce processing costs, wealth storage \& micro-payments require proof-of-stake consensus mechanisms along with public unpermissioned blockchains and pseudonymous users. Public permissioned blockchains with some modifications of proof-ofwork consensus mechanism support financial services by expanding the functionality of payments through financial checks and deposits. Energyefficient financial services use blockchains with the same characteristics as financial services except for proof-of-stake consensus mechanisms. Enterprise global- and micro financial transactions employ private unpermissioned blockchains with practical Byzantine fault tolerance consensus mechanism, which requires unique identification of nodes in the network. Global centrally issued financial instruments are deployed on private permissioned blockchains with self-developed consensus mechanisms, which also require unique identification of the nodes.

The second application area is smart contracts and processes application logic. The application area contains eight application cases. Most smart contracts work on public unpermissioned blockchains with a proof-of-work consensus mechanism. At the same time, a proof-of-stake consensus mechanism supports energy-efficient smart contracts. For testing purposes, one can create private blockchains that comprise only one node. Community smart contracts, which must comply with different community rules, are based on public permissioned blockchains with proof-of-work consensus mechanisms. Energy-efficient community smart contracts apply proof-of-stake consensus mechanisms. Enterprise smart contracts use private unpermissioned blockchains. Global agreements between institutions can be achieved based on private permissioned blockchains.

The third application area is data management and is concerned with information management, such as authentication, know-your-customer services, and control of business assets. The area includes three application cases. To manage assets registered offchain, global authentication and ownership require public unpermissioned blockchains with proof-ofwork consensus mechanisms and pseudonymous users. Sharing economies and enterprise asset management require data management with identification and authorization schemes implemented directly on a blockchain. To avoid fraud although opening a network for many nodes, sharing economies use public permissioned blockchains with proof-of-work consensus mechanisms and identifiable users. To keep information confidential, enterprise asset management applies private permissioned blockchains that reach system-level consensus by practical Byzantine fault tolerance and require unique identification of nodes.

The fourth application area is storage and is concerned with keeping digital assets, such as certificates or music and video files, on blockchains. Open access publishing uses public blockchains and requires no data encryption. Content preview employs public blockchains with partial encryption of data. Blockchain-based decentralized storage is implemented on public blockchains with total data encryption and some modifications for faster content sharing and decoding.

The fifth application area is communication. Broadcasting is supported by public unpermissioned blockchains with proof-of-work consensus mechanisms and without data encryption because the content is intended for mass communication. Public permissioned blockchains with proof-of-work consensus mechanisms are suitable for discussion forums, which allow any user to participate in communication but automatically collect IP addresses. Internet-of-things communication uses private unpermissioned blockchains and practical Byzantine fault tolerance consensus mechanism to control information exchange between devices in enterprise or home networks.

The sixth application area is ranking with a single application case. Global reputation \& rating is supported by public permissioned blockchain with proof-of-work consensus mechanisms and automatic collection of identifiers to link identities to individual users and to prevent users from obtaining more than one identity.

\subsection{Demonstration of the utility of the taxonomy}

We demonstrate the utility of the taxonomy on ninety-nine blockchain-based systems mentioned in the scientific and business sources. To classify identified blockchain-based systems with the taxonomy, we used white papers, the systems' websites, press releases, and set up the systems and tested them if it was possible. The demonstration of the utility of the taxonomy shows that the taxonomy classifies successful blockchain-based systems and purposefully does not classify some blockchain-based systems.

4.3.1. Classified blockchain-based systems. The gathered blockchain-based systems predominantly cover the financial sector. Anonymous cryptocurrencies include Zerocoin, Darkcoin, 
CryptoNote, and Monero. Conventional cryptocurrencies comprise Bitcoin, Primecoin, Litecoin, Tether, DagCoin, Crypt Cryptosigma, DigixGlobal, GameCredits, Bitpay, and SolarCoin. Peercoin, Navcoin, AML, and Blackcoin target wealth storage \& micro-payments. Counterparty, Mastercoin, and DigitalNote execute financials services. BitShares allows energy-efficient financial services. Ripple, SWIFT gpi, Stellar, and BitPesa support enterprise global and micro- financial transactions. R3, Fedcoin, Symbiont Assembly, RSCoin, and Onecoin represent global centrally issued financial instruments.

Smart contracts are popular for the identified blockchain-based systems. Ethereum, Hawk, Stratis, Qtum, Blockcypher, Deckbound, Rootstock, iExec, Chimera, WeTrust, Sia, and Maidsafe support original smart contracts. Testing of smart contracts is

Table 1. A taxonomy of blockchain applications.

\begin{tabular}{|c|c|c|c|c|c|c|c|c|c|c|c|c|c|c|c|c|c|c|c|c|c|c|}
\hline \multirow{2}{*}{\multicolumn{2}{|c|}{$\begin{array}{c}\text { A TAXONOMY OF BLOCKCHAIN } \\
\text { APPLICATIONS }\end{array}$}} & \multicolumn{2}{|c|}{$\begin{array}{l}\text { Reading } \\
\text { access }\end{array}$} & \multicolumn{2}{|c|}{$\begin{array}{l}\text { Writing } \\
\text { access }\end{array}$} & \multicolumn{4}{|c|}{$\begin{array}{l}\text { Main consensus } \\
\text { mechanism }\end{array}$} & \multicolumn{3}{|c|}{$\begin{array}{c}\text { Anonymity } \\
\text { level }\end{array}$} & \multicolumn{3}{|c|}{$\begin{array}{l}\text { Event } \\
\text { handling }\end{array}$} & \multicolumn{2}{|c|}{$\begin{array}{l}\text { Data } \\
\text { exchange } \\
\text { type }\end{array}$} & \multicolumn{3}{|c|}{ Encryption } & \multicolumn{2}{|c|}{$\begin{array}{l}\text { History } \\
\text { retention }\end{array}$} \\
\hline & & $\operatorname{Pr}$ & $\mathrm{Pu}$ & $\mathrm{P}$ & U & $\mathrm{W}$ & S & B & SD & A & $\mathrm{P}$ & I & $\mathrm{N}$ & $\mathrm{F}$ & $\mathrm{C}$ & $\mathrm{T}$ & $\mathrm{C}$ & $\mathrm{U}$ & $\mathrm{P}$ & $\mathrm{T}$ & W & $\mathrm{R}$ \\
\hline \multirow{7}{*}{$\begin{array}{l}\text { Financial } \\
\text { transactions }\end{array}$} & $\begin{array}{l}\text { Anonymous } \\
\text { cryptocurrencies }\end{array}$ & & $\mathrm{X}$ & & $\mathrm{X}$ & $\mathrm{X}$ & & & & $\mathrm{X}$ & & & $\mathrm{X}$ & & & $\mathrm{X}$ & & & & $\mathrm{X}$ & $\mathrm{X}$ & \\
\hline & Cryptocurrencies & & $X$ & & $\mathrm{X}$ & $\mathrm{X}$ & & & & & $\mathrm{X}$ & & $X$ & & & $X$ & & $\mathrm{X}$ & & & $X$ & \\
\hline & $\begin{array}{l}\text { Wealth storage \& } \\
\text { micro-payments }\end{array}$ & & $\mathrm{X}$ & & $\mathrm{X}$ & & $\mathrm{X}$ & & & & $\mathrm{X}$ & & $\mathrm{X}$ & & & $\mathrm{X}$ & & $\mathrm{X}$ & & & $\mathrm{X}$ & \\
\hline & Financial services & & $\mathrm{X}$ & $\mathrm{X}$ & & $\mathrm{X}$ & & & & & $X$ & & $X$ & & & $X$ & & $\mathrm{X}$ & & & $\mathrm{X}$ & \\
\hline & $\begin{array}{l}\text { Energy-efficient } \\
\text { financial services }\end{array}$ & & $\mathrm{X}$ & $\mathrm{X}$ & & & $\mathrm{X}$ & & & & $\mathrm{X}$ & & $\mathrm{X}$ & & & $\mathrm{X}$ & & $\mathrm{X}$ & & & $\mathrm{X}$ & \\
\hline & $\begin{array}{l}\text { Enterprise global } \\
\text { and micro- } \\
\text { financial } \\
\text { transactions }\end{array}$ & $\mathrm{X}$ & & & $\mathrm{X}$ & & & $X$ & & & & $\mathrm{X}$ & $\mathrm{X}$ & & & $\mathrm{X}$ & & & $\mathrm{X}$ & & $\mathrm{X}$ & \\
\hline & $\begin{array}{l}\text { Global centrally } \\
\text { issued financial } \\
\text { instruments }\end{array}$ & $\mathrm{X}$ & & $X$ & & & & & $X$ & & & $\mathrm{X}$ & $\mathrm{X}$ & & & $\mathrm{X}$ & & & $\mathrm{X}$ & & $\mathrm{X}$ & \\
\hline \multirow{8}{*}{ Smart contracts } & Smart contracts & & $\mathrm{X}$ & & $\mathrm{X}$ & $\mathrm{X}$ & & & & & $\mathrm{X}$ & & & & $\mathrm{X}$ & $\mathrm{X}$ & & $\mathrm{X}$ & & & $\mathrm{X}$ & \\
\hline & $\begin{array}{l}\text { Testing of smart } \\
\text { contracts }\end{array}$ & $\mathrm{X}$ & & & $\mathrm{X}$ & $\mathrm{X}$ & & & & & $\mathrm{X}$ & & & & $X$ & $\mathrm{X}$ & & $\mathrm{X}$ & & & $\mathrm{X}$ & \\
\hline & $\begin{array}{l}\text { Energy-efficient } \\
\text { smart contracts }\end{array}$ & & $\mathrm{X}$ & & $\mathrm{X}$ & & $\mathrm{X}$ & & & & $\mathrm{X}$ & & & & $\mathrm{X}$ & $\mathrm{X}$ & & $\mathrm{X}$ & & & $\mathrm{X}$ & \\
\hline & $\begin{array}{l}\text { Testing of } \\
\text { energy-efficient } \\
\text { smart contracts }\end{array}$ & $\mathrm{X}$ & & & $\mathrm{X}$ & & $\mathrm{X}$ & & & & $\mathrm{X}$ & & & & $\mathrm{X}$ & $\mathrm{X}$ & & $\mathrm{X}$ & & & $\mathrm{X}$ & \\
\hline & $\begin{array}{l}\text { Community } \\
\text { smart contracts }\end{array}$ & & $\mathrm{X}$ & $\mathrm{X}$ & & $\mathrm{X}$ & & & & & $\mathrm{X}$ & & & & $\mathrm{X}$ & $\mathrm{X}$ & & $\mathrm{X}$ & & & $X$ & \\
\hline & $\begin{array}{l}\text { Energy-efficient } \\
\text { community smart } \\
\text { contracts }\end{array}$ & & $\mathrm{X}$ & $X$ & & & $\mathrm{X}$ & & & & $X$ & & & & $\mathrm{X}$ & $X$ & & $\mathrm{X}$ & & & $\mathrm{X}$ & \\
\hline & $\begin{array}{l}\text { Enterprise smart } \\
\text { contracts }\end{array}$ & $\mathrm{X}$ & & & $\mathrm{X}$ & & & $\mathrm{X}$ & & & & $\mathrm{X}$ & & & $\mathrm{X}$ & $\mathrm{X}$ & & & $\mathrm{X}$ & & $X$ & \\
\hline & $\begin{array}{l}\text { Global } \\
\text { agreements } \\
\text { between } \\
\text { institutions }\end{array}$ & $\mathrm{X}$ & & $\mathrm{X}$ & & & & & $\mathrm{X}$ & & & $\mathrm{X}$ & & & $\mathrm{X}$ & $\mathrm{X}$ & & & $\mathrm{X}$ & & $\mathrm{X}$ & \\
\hline \multirow{3}{*}{ Data management } & $\begin{array}{l}\text { Global } \\
\text { authentication } \\
\text { and ownership }\end{array}$ & & $X$ & & $\mathrm{X}$ & $\mathrm{X}$ & & & & & $\mathrm{X}$ & & & $\mathrm{X}$ & & $\mathrm{X}$ & & $\mathrm{X}$ & & & $\mathrm{X}$ & \\
\hline & $\begin{array}{l}\text { Sharing } \\
\text { economies }\end{array}$ & & $\mathrm{X}$ & $\mathrm{X}$ & & $\mathrm{X}$ & & & & & & $\mathrm{X}$ & & $\mathrm{X}$ & & $\mathrm{X}$ & & $\mathrm{X}$ & & & $\mathrm{X}$ & \\
\hline & $\begin{array}{l}\text { Enterprise asset } \\
\text { management }\end{array}$ & $\mathrm{X}$ & & & $\mathrm{X}$ & & & $\mathrm{X}$ & & & & $\mathrm{X}$ & & $\mathrm{X}$ & & $\mathrm{X}$ & & & $\mathrm{X}$ & & $\mathrm{X}$ & \\
\hline \multirow{3}{*}{ Storage } & $\begin{array}{l}\text { Open access } \\
\text { publishing }\end{array}$ & & $\mathrm{X}$ & $\mathrm{X}$ & & $\mathrm{X}$ & & & & & & $\mathrm{X}$ & & $\mathrm{X}$ & & & $\mathrm{X}$ & $\mathrm{X}$ & & & $\mathrm{X}$ & \\
\hline & Content preview & & $X$ & $X$ & & $\mathrm{X}$ & & & & & & $\mathrm{X}$ & & $\mathrm{X}$ & & & $\mathrm{X}$ & & $\mathrm{X}$ & & $\mathrm{X}$ & \\
\hline & $\begin{array}{l}\text { Decentralized } \\
\text { storage }\end{array}$ & & $\mathrm{X}$ & $\mathrm{X}$ & & $\mathrm{X}$ & & & & & & $\mathrm{X}$ & & $\mathrm{X}$ & & & $\mathrm{X}$ & & & $\mathrm{X}$ & $\mathrm{X}$ & \\
\hline \multirow{3}{*}{ Communication } & Broadcasting & & $\mathrm{X}$ & & $\mathrm{X}$ & $\mathrm{X}$ & & & & & & $\mathrm{X}$ & & $\mathrm{X}$ & & & $\mathrm{X}$ & $\mathrm{X}$ & & & & $\mathrm{X}$ \\
\hline & $\begin{array}{l}\text { Discussion } \\
\text { Forum }\end{array}$ & & $X$ & $\mathrm{X}$ & & $\mathrm{X}$ & & & & & & $\mathrm{X}$ & & $\mathrm{X}$ & & & $\mathrm{X}$ & $\mathrm{X}$ & & & & $\mathrm{X}$ \\
\hline & $\begin{array}{l}\text { IoT } \\
\text { communication }\end{array}$ & $\mathrm{X}$ & & & $\mathrm{X}$ & & & $\mathrm{X}$ & & & & $\mathrm{X}$ & & $\mathrm{X}$ & & & $\mathrm{X}$ & $\mathrm{X}$ & & & & $\mathrm{X}$ \\
\hline Ranking & $\begin{array}{l}\text { Global reputation } \\
\& \text { rating }\end{array}$ & & $\mathrm{X}$ & $\mathrm{X}$ & & $\mathrm{X}$ & & & & & & $\mathrm{X}$ & & $\mathrm{X}$ & & $\mathrm{X}$ & & $\mathrm{X}$ & & & & $\mathrm{X}$ \\
\hline \multicolumn{10}{|c|}{$\begin{array}{l}\text { LEGEND } \\
\text { X - characteristics belong to an application case } \\
\text { Reading access } \\
\text { Pr - Private: only authorized members of a limited community can read blockchain } \\
\text { Pu - Public: everybody can read a blockchain } \\
\text { Writing access } \\
\text { P - Permissioned: a user should be authorized to validate transactions } \\
\text { U - - Unpermissioned: a user can validate transactions without authorization } \\
\text { Main consensus mechanism } \\
\text { W - Proof-of-work: consensus for secure blockchain updating is achieved by Proof-of-Work } \\
\text { S - Proof-of-stake: consensus for secure blockchain updating is achieved by Proof-of-Stake } \\
\text { B - Practical Byzantine fault tolerance: secure blockchain updating is achieved by } \\
\text { agreements of trusted nodes } \\
\text { SD - Self-developed mechanism: consensus for secure blockchain updating is achieved by } \\
\text { self-developed mechanism } \\
\text { Anonymity level } \\
\text { A - Anonymous: users do not have to provide any data for working with blockchain } \\
\text { P - Pseudonymous: users can work with a blockchain under a pseudonym } \\
\text { I - Identifiable: users should provide personal data to work with a blockchain }\end{array}$} & \multicolumn{13}{|c|}{$\begin{array}{l}\text { Event handling } \\
\text { No - No: blockchain does not support any events } \\
\text { F - Fixed: blockchain supports built-in events } \\
\text { C - } \text { Custom: blockchain supports processing of events created by user } \\
\text { Data exchange type } \\
\text { T - Transaction: logs of actions executed are exchanged among users and } \\
\text { recorded on a blockchain } \\
\text { C - Content: digital assets are exchanged among users and recorded on a } \\
\text { blockchain } \\
\text { Encryption } \\
\text { U - Unencrypted: all data on a blockchain is unencrypted } \\
\text { P - Partially-encrypted: data on a blockchain is partially encrypted } \\
\text { T - Totally-encrypted: all data on a blockchain is encrypted } \\
\text { History retention } \\
\text { W - Whole: blockchain keeps whole transaction history from a genesis } \\
\text { block } \\
\mathrm{R}-\text { Recent updates: blockchain keeps only recent updates of the transaction } \\
\text { history }\end{array}$} \\
\hline
\end{tabular}


possible on Ethereum (testing environment), Hawk (testing environment), and EOS. Casper, Tendermint, and Nxt develop energy-efficient smart contracts. Testing of energy-efficient smart contracts is performed on Casper (testing environment). Counterparty supports community smart contracts. Lisk and Tezos execute energy-efficient community smart contracts. Hyperledger, Ripple Codius, Eris (Monax), Digital asset, Waves, and Catenis Enterprise support enterprise smart contracts. R3 Codra allows reaching global agreements between institutions.

Data management on blockchains gains momentum. Colored coins, Namecoin, Onename, POEX.IO, OP_RETURN, Everpass, The Real McCoy, BitHealth, BitAuth, UniquID, NEM Apostille, Blockname, Filament, ePlug, and Shocard represent global authentication and ownership. Iconomi, NEO, Ridde \& code, Aragon, and La'Zooz are examples of sharing economies. Everledger, PeerNova, Factom, Chromaway, BlockVerify, PeerNova, Chronicled, and ShoBadge support enterprise asset management.

A smaller number of blockchain applications supports blockchain-based storage. Synereo fulfills open access publishing. Kishigami et al. [22] describe content preview on blockchains; although we did not find blockchain-based systems to support the application case, we decided to keep the application case for further research. The Storj project examines decentralized storage on blockchain.

Communication is not often implemented on blockchains. Basic Attention Token shows broadcasting. Blockchain-based discussion forums include Whisper and Matchpool. Blockchain of Things and IBM Adept support internet-of-things communication.

Ranking on blockchains is an uncommon blockchain application case. Augur, TRST.im, The World Table, and TrustDavis support global reputation \& rating.

4.3.2. Unclassified blockchain-based systems. We found blockchain-based systems that purposefully remain unclassified by our taxonomy. The first reason for unclassified blockchain-based systems is an application area that appears to be unsuitable for blockchains. Such blockchain applications have broad ideas and aim to replace current information systems with blockchains (e.g., decentralized internet); however, they do not result in any proofs of concept. Other examples arise when blockchain applications use blockchains when blockchains are not needed (e.g., private messengers on blockchains can be replaced by conventional peer-to-peer systems).

The second reason of unclassified blockchainbased systems is combinations of technical blockchain characteristics that appear to be ineffective. These blockchain-based systems exhibit or intensify security threats or privacy concerns. For example, hackers attack blockchains by forking them, developers of blockchain-based systems can falsify data on blockchains, and users can be traceable when permissions to read and write data on blockchains do not comply with consensus mechanisms or with anonymity protection of users.

The third reason of unclassified blockchain-based systems is a combination of blockchain application areas and technical blockchain characteristics that appear to be unsuitable. For example, a blockchainbased system that aims to manage certificates between trustful organizations (e.g., school diplomas between schools and employee companies) is an example of enterprise asset management. However, an application we identified uses a public blockchain with a proof-of-work consensus mechanism instead of a private blockchain with a practical Byzantine fault tolerance consensus mechanism. The reason why the application uses a blockchain is not due to the actual number of nodes but due to the borrowed public infrastructure. The following concerns arise. If the application uses a public blockchain, transactions are expensive because of the consensus mechanism. For transactions on this blockchain the issuers of the certificates (e.g., schools) must be trustful to prevent information manipulation or fraud (e.g., an actor could send transactions to himself to change records). However, if issuers are trustful, a public blockchain is useless. Therefore, the blockchain application ignores the main dilemma in using blockchains and public-private infrastructure: the more trustful issuers are, the less energy-consuming the employed consensus mechanism should be.

\section{Discussion}

The developed taxonomy serves as a bridge between blockchain technology and blockchain application areas. The taxonomy constitutes a tool to connect technical blockchain characteristics across a range of foundational application cases. There are five principal findings. First, application areas in the taxonomy are at different maturity levels. Financial transactions constitutes the most mature application area and is supported by existing proofs of concept. Smart contracts have found much attention because of the idea to execute agreements on blockchains instead of third parties. Data management gains 
momentum because of emerging application cases (e.g., enterprise asset management on private blockchains). Storage, communication, and ranking on blockchains are less prevalent. Blockchain scalability issues prevent storage of data on blockchains. The value of applying blockchains for communication and ranking is specific to each application case. In particular, it is challenging to support mobile devices when energy-consuming consensus mechanisms and the transfer of the whole transaction history are required.

Second, application cases inside one application area vary in the dimensions reading access, writing access, main consensus mechanism, and anonymity level. The characteristics in these dimensions depend on the required levels of decentralization for application cases. The more centralization is required, the more private reading access and the more permissioned writing access is required. Main consensus mechanism and anonymity level follow the required level of decentralization so that the more centralization is required, the less energy-consuming are consensus mechanisms and the less anonymous are nodes.

Third, to classify application areas, we reveal new technical dimensions that are overlooked in extant technical classifications on blockchains due to its predominant focus on the financial sector. The new dimensions are event handling, data exchange type, encryption, and history retention. Custom event handling specifies smart contracts. Data exchange type allocates whether data is stored on or off blockchains. Encryption is different between application cases that require to store content or transactions on blockchains. History retention is different for application cases that store blockchains on small-capacity external devices and have to remove old information from blockchains.

Fourth, not all and different technical blockchain characteristics are suitable for different application areas. For example, communication systems based on private permissioned blockchains do not appear to create additional value compared to peer-to-peer messengers such as Telehash, which are used by many decentralized services (e.g., IBM Adept). However, this statement requires further investigation.

Fifth, the taxonomy purposefully avoids the classification of poorly developed blockchain-based systems because blockchain application cases are identified and related to unique and effective combinations of technical characteristics. Therefore, blockchain-based systems that are not captured by the taxonomy might represent application areas that are unsuitable for blockchains. Combinations of technical characteristics that contradict the taxonomy can lead to inefficient technical designs. Inconsistencies between application areas and technical designs may indicate a lack of compliance with technical and application requirements. However, the taxonomy is only based on extant knowledge in research and practice and this assertion requires further research.

There are three promising areas for future research. First, research that replicates our research approach with more or different scientific and business sources will be useful to falsify or corroborate our findings. Second, further analysis of theoretical findings allows to hypothesize about the relationships between application areas and technical blockchain characteristics. Third, research that focuses on socio-economic concepts different from application areas, for example, market regulations in different countries will be useful to contextualize the taxonomy for different industries and domains.

This study is not without limitations. First, the taxonomy cannot identify application areas that may emerge in the future. The rapidly evolving nature of the blockchain domain will necessitate an extension of the taxonomy with new application cases. Second, the identified application areas do not directly capture more complex services, such as prediction markets or crowdsourcing platforms; instead, we decided to break complex application cases down into the basic functionalities that can be performed by blockchains.

This research contributes to the scientific literature on blockchain in three ways. First, allocation of blockchain application cases based on technical blockchain characteristics reduces the hype around blockchain application possibilities. A classification of application areas that, along with semantic differences, is based on technical characteristics make the identification of application areas more meaningful. The well-studied financial sector can serve as a good example for how to leverage blockchains in less studied application areas and the other application areas may reveal opportunities that have been overlooked in the financial sector. Second, we identified additional technical dimensions of importance in the blockchain domain. While some of the taxonomy dimensions (reading access, writing accesses, main consensus mechanisms, and anonymity level) align with previous taxonomies, the remaining dimensions (event handling, data exchange type, encryption, and history retention) represent specific application areas and complement previous taxonomies by offering more comprehensive insights into the technical nature of blockchains. Therefore, technical research can go beyond the Bitcoin blockchain and focus on 
other areas, for example, development of a blockchain-based protocol for data transmission in healthcare. Third, previous taxonomies consider technical knowledge [17] or application knowledge [12] separately. Our taxonomy combines the knowledge, which allows to bridge the gap between extant technical and application research streams on blockchain. Linking application areas and technical characteristics informs step-by-step guidelines for leveraging blockchains across application areas. Such guidelines might be useful for further development of successful blockchain-based systems.

This research contributes to practice in three ways. First, we present further evidence that blockchains are not only applicable to the financial sector, which is the focus of the majority of blockchain projects but also for other promising areas. Thus, other industries can use blockchain advantages for resolving their challenges. For example, in the media industry blockchain-based data management may be useful to monitor the use of media content to prevent copyright infringements. Second, we highlight other blockchain characteristics besides the widely-known public blockchains that can be useful if public blockchains cannot be employed. Businesses may consider implementation of private blockchains that store information in a more reliable way. Third, we have proposed the taxonomy of blockchain applications to guide development of more successful blockchain-based systems. The taxonomy establishes an overview of blockchain applications, organizes them in application areas, and relates them to technical blockchain characteristics. Furthermore, the taxonomy can be used to avoid poorly designed blockchain applications. This might be useful for practitioners to identify the more promising blockchain projects and assess risks during blockchain implementation. For example, chief information officers could learn which modules in the enterprise information systems landscape can be realized on blockchains and developers could learn which peer-to-peer system prototypes are worth to be develop on blockchains.

\section{Conclusion}

A blockchain is a decentralized digital ledger with a largely untapped potential to enhance many aspects in the information systems domain. Currently, research streams on blockchain remain disconnected, which prevents further development of successful blockchain-based systems. Our work consolidates knowledge on technical blockchain characteristics and application areas in the form of a taxonomy. The taxonomy accounts for twenty-five application cases aggregated into six application areas that relate to twenty-one technical blockchain characteristics in eight dimensions. Overall, the taxonomy consolidates extant knowledge on blockchains to calm the blockchain hype and foster development of more realistic blockchain-based systems.

\section{References}

[1] Avital, M., J.L. King, R. Beck, M. Rossi, and R. Teigland, "Jumping on the Blockchain Bandwagon : Lessons of the Past and Outlook to the Future Panel", Thirty Seventh International Conference on Information Systems, (2016), 1-6.

[2] Azaria, A., A. Ekblaw, T. Vieira, and A. Lippman, "MedRec: Using Blockchain for Medical Data Access and Permission Management", 2nd International Conference on Open and Big Data (OBD), (2016), 25-30.

[3] Beck, R., and C. Müller-Bloch, "Blockchain as Radical Innovation: A Framework for Engaging with Distributed Ledgers as Incumbent Organization”, 50th Hawaii International Conference on System Sciences, (2017), 5390-5399.

[4] Bloom, B., "Bloom 's Taxonomy”, ReVision, 2001, pp. $1-6$.

[5] Böhm, M., J. Weking, F. Fortunat, S. Müller, and I.

Welpe, "The Business Model DNA : Towards an Approach for Predicting Business Model Success", 13th International Conference on Wirtschaftsinformatik, (2017), 9-11.

[6] Bonneau, J., A. Miller, J. Clark, A. Narayanan, J.A. Kroll, and E.W. Felten, "Research Perspectives and Challenges for Bitcoin and Cryptocurrencies", IEEE Symposium on Security and Privacy, 2015, pp. 104-121. [7] vom Brocke, J., A. Simons, B. Niehaves, et al., "Reconstructing the Giant: On the Importance of Rigour in Documenting the Literature Search Process", 17th

European Conference on Information Systems 9, 2009, pp. 2206-2217.

[8] Condos, J., W.H. Sorrell, and S.L. Donegan, "Blockchain Technology: Opportunities and Risks", Vermont(Vermont), 2016, pp. 1-35.

[9] Constantinides, P., O. Henfridsson, and G. Parker, "Platforms and Infrastructures in the Digital Age", Information Systems Research(May), 2018, pp. 1-20. [10] Darwin, C., "The Origin of Species (1859)", In Foundations of Psychological Thought: A History of Psychology. 2009, 320-336.

[11] Fabian, B., T. Ermakova, and U. Sander, "Anonymity in Bitcoin - The Users' Perspective", 37th International Conference on Information Systems (ICIS 2016), (2016), 112.

[12] Friedlmaier, M., A. Tumasjan, and I.M. Welpe, "Disrupting Industries With Blockchain: The Industry, Venture Capital Funding, and Regional Distribution of Blockchain Ventures", SSRN Electronic Journal, 2016. [13] Furlonger, D., and R. Valdes, Practical Blockchain: A Gartner Trend Insight Report, 2017.

[14] Garman, C., M. Green, I. Miers, and A.D. Rubin, 
"Rational Zero: Economic Security for Zerocoin with Everlasting Anonymity", International Conference on Financial Cryptography and Data Security, Springer, Berlin, Heidelberg, 140-155.

[15] Gibbs, T., and S. Yordchim, "Thai Perception on Litecoin Value", International Journal of Social, Behavioral, Educational, Economic, Business and Industrial Engineering 8(8), 2014, pp. 2613-2615. [16] Glaser, F., "Pervasive Decentralisation of Digital Infrastructures: A Framework for Blockchain enabled System and Use Case Analysis", 50th Hawaii International Conference on System Sciences, (2017), 1543-1552. [17] Glaser, F., and L. Bezzenberger, "Beyond Cryptocurrencies - A Taxonomy of Decentralized Consensus Systems", Proceedings of the 23rd European Conference on Information Systems (ECIS 2015), (2015), $1-18$.

[18] Higgins, S., "Factom Partners With Honduras Government on Blockchain Tech Trial", CoinDesk, 2015. https://www.coindesk.com/factom-land-registry-dealhonduran-government/ [19] Hyperledger Architecture Working Group, "Hyperledger Architecture, Volume 1", Hyperledger.org 1, 2017, pp. 15.

[20] Kannengießer, N., S. Lins, T. Dehling, and A. Sunyaev, "What Does Not Fit Can be Made to Fit! TradeOffs in Distributed Ledger Technology Designs", Proceedings of the 52nd Hawaii International Conference on System Sciences, (2019).

[21] Kazan, E., C. Tan, and E.T.K. Lim, "Towards a Framework of Digital Platform Disruption : A Comparative Study of Centralized \& Decentralized Digital Payment Providers", 25th Australasian Conference on Information Systems(2010), 2014, pp. 10.

[22] Kishigami, J., S. Fujimura, H. Watanabe, A. Nakadaira, and A. Akutsu, "The Blockchain-Based Digital Content Distribution System”, 2015 IEEE Fifth

International Conference on Big Data and Cloud Computing, (2015), 187-190.

[23] Morisse, M., "Cryptocurrencies and Bitcoin: Charting the Research Landscape", Americas Conference on Information Systems, 2015.

[24] Mrosek, R., T. Dehling, and A. Sunyaev, "Taxonomy of Health IT and Medication Adherence", Health Policy and Technology 4(3), 2015, pp. 215-224.

[25] Nakamoto, S., "Bitcoin: A Peer-to-Peer Electronic Cash System", Www.Bitcoin.Org, 2008, pp. 9.

[26] Nickerson, R.C., U. Varshney, and J. Muntermann, “A Method for Taxonomy Development and its Application in Information Systems", European Journal of Information Systems 22(3), 2013, pp. 336-359.

[27] Pongnumkul, S., C. Siripanpornchana, and S. Thajchayapong, "Performance Analysis of Private Blockchain Platforms in Varying Workloads", 2017 26th International Conference on Computer Communications and Networks, ICCCN 2017, (2017).

[28] Pureswaran, V., S. Panikkar, S. Nair, and P. Brody, Empowering the Edge: Practical Insights on a Decentralized Internet of Things, 2015.

[29] Reid, F., and M. Harrigan, "An Analysis of Anonimity in the Bitcoin System", IEEE International Conference on
Privacy, Security, Risk, and Trust, and IEEE International Conference on Social Computing, (2011), 1318-1326.

[30] Salviotti, G., L.M. De Rossi, and N. Abbatemarco, “A Structured Framework to Assess the Business Application Landscape of Blockchain Technologies", Proceedings of the 51st Hawaii International Conference on System Sciences, (2018), 3467-3476.

[31] Schneider, S., J. Lansing, F. Gao, and A. Sunyaev, “A Taxonomic Perspective on Certification Schemes: Development of a Taxonomy for Cloud Service Certification Criteria", Proceedings of the Annual Hawaii International Conference on System Sciences, (2014), 4998-5007.

[32] Sharples, M., and J. Domingue, "The Blockchain and Kudos: A Distributed System for Educational Record, Reputation and Reward", Lecture Notes in Computer Science (including subseries Lecture Notes in Artificial Intelligence and Lecture Notes in Bioinformatics), (2016). [33] Strauss, A., and J. Corbin, Strauss, A., \& Corbin, J. (1990)., 2008.

[34] Strauss, A.L., Qualitative Analysis for Social Scientists, 1987.

[35] Swan, M., "Blockchain Thinking : the Brain as a Decentralized Autonomous Corporation [Commentary]", IEEE Technology and Society Magazine 34(4), 2015, pp. 41-52.

[36] Swan, M., Blockchain: Blueprint for a New Economy, 2015.

[37] The Economist, "The Trust Machine - The promise of the blockchain", The Economist, 2015, 2-4.

[38] Tschorsch, F., and B. Scheuermann, "Bitcoin and Beyond: A Technical Survey on Decentralized Digital Currencies", IEEE Communications Surveys and Tutorials, (2016).

[39] Walsh, C., P. O’Reilly, R. Gleasure, J. Feller, L. Shanping, and J. Cristoforo, "New Kid on the Block: a Strategic Archetypes Approach to Understanding the Blockchain", 37th International Conference on Information Systems (ICIS), (2016), 1-12.

[40] Webster, J., and R.T. Watson, "Analyzing the Past to Prepare for the Future: Writing a Literature Review.", MIS Quarterly 26(2), 2002, pp. xiii-xxiii.

[41] Welpe, I., L. Zavolokina, H. Krcmar, and M. Müller, "Introduction to Distributed Ledger Technology, Blockchain Minitrack", (2018), 3465-3466.

[42] Wiesche, M., M.C. Jurisch, P.W. Yetton, and H. Krcmar, "Grounded Theory Methodology in Information Systems Research", MIS Quarterly 41(3), 2017, pp. 685A9.

[43] Wörner, D., T. Von Bomhard, Y.-P. Schreier, and D. Bilgeri, "The Bitcoin Ecosystem: Disruption beyond financial Services?", Twenty-Fourth European Conference on Information Systems (ECIS), (2016), 1-16.

[44] Xu, X., I. Weber, M. Staples, et al., "A Taxonomy of Blockchain-Based Systems for Architecture Design", Proceedings - 2017 IEEE International Conference on Software Architecture, ICSA 2017, (2017), 243-252. [45] Yli-Huumo, J., D. Ko, S. Choi, S. Park, and K. Smolander, "Where is Current Research on Blockchain Technology? - A Systematic Review", PLoS ONE 11(10), 2016, pp. 1-27. 